\title{
Cannabis and epilepsy
}

\author{
Rhys H Thomas, ${ }^{1}$ Mark O Cunningham ${ }^{2}$
}

${ }^{1}$ Institute of Neuroscience, Newcastle University, Royal Victoria Infirmary, Newcastleupon-Tyne, UK

${ }^{2}$ School of Medicine, Discipline of Physiology, University of Dublin Trinity College, Dublin, Ireland

\section{Correspondence to}

Rhys $\mathrm{H}$ Thomas, Institute of Neuroscience, Newcastle University, Royal Victoria Infirmary, Newcastle-upon-Tyne NE2 4HH, UK; Rhys.Thomas@ newcastle.ac.uk

Accepted 23 September 2018 Published Online First

18 October 2018

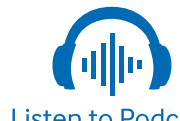

Listen to Podcast pn.bmj.com

\section{Check for updates}

(c) Author(s) (or their employer(s)) 2018. No commercial re-use. See rights and permissions. Published by BMJ.

To cite: Thomas $\mathrm{RH}$, Cunningham MO. Pract Neurol 2018:18:465-471.

\begin{abstract}
The one-third of people who do not gain seizure control through current treatment options need a revolution in epilepsy therapeutics. The general population appears to be showing a fundamental and rapid shift in its opinion regarding cannabis and cannabis-related drugs. It is quite possible that cannabidiol, licensed in the USA for treating rare genetic epilepsies, may open the door for the widespread legalisation of recreational cannabis. It is important that neurologists understand the difference between artisanal cannabidiol products available legally on the high street and the cannabidiol medications that have strong trial evidence. In the UK in 2018 there are multiple high-profile reports of the response of children taking cannabisderived medication, meaning that neurologists are commonly asked questions about these treatments in clinic. We address what an adult neurologist needs to know now, ahead of the likely licensing of Epidiolex in the UK in 2019.
\end{abstract}

\section{INTRODUCTION}

We have seen a surge in interest in the medicinal use of cannabis and cannabis extracts for people with epilepsy. Historically, there are reports from ancient and medieval times of using cannabis to treat epilepsy. During the 19th century, the introduction of medicinal cannabis into Western medicine was driven by the experiences and studies of William O'Shaughnessy, from his time working in India with the East India Company. His publications led to the acceptance of the use of cannabis in Victorian England. Indeed, two distinguished neurologists of that era, J.R. Reynolds and William Gowers, documented their experiences with cannabis, particularly for the treatment of epilepsy. However, in the 20th century, fluctuating public morals and worries about cannabis' psychoactive properties triggered the global outlawing of cannabis.

In 2018 there have both been developments in class I evidence from randomised controlled trials and a growing momentum of patient stories based on lived experiences. In June 2018 the US Food and
Drug Administration (FDA) approved Epidiolex, a cannabidiol (CBD) oral solution for use in Lennox-Gastaut and Dravet syndrome for patients aged $\geq 2$ years. ${ }^{1}$ This is a landmark as it represents not only the first FDA approval for a drug specifically trialled in Dravet syndrome but also the first drug that contains a purified substance derived from marijuana.

Cannabis-derived drugs are very different from cannabis that is available legally or illegally, in terms of the reliability of their content and purity; and cannabis-derived medications differ from CBD oils. The drugs are either extracted from plants-such as Epidiolex, the CBD is a cannabinoid prepared from the Cannabis sativa L. plant-or they are synthesised. CBD oils vary greatly, and commercial CBD oil sites trumpet this impurity as a benefit: such as the claim by 'Charlotte's Web' 'Counter normal, everyday stresses with the goodness of nature. Cannabidiol (CBD) plus other naturally occurring phytocannabinoids, terpenes, flavonoids and more in every serving'.

The number of active compounds within cannabis is debated but tetrahydrocannabinol (THC), CBD and cannabidivarin are but three of approximately 85 known. The available pharmaceutical drugs are a balance between CBD and THC; the more THC that a drug contains, the more psychoactive it is likely to be, producing either a 'high' or a calming effect. In general, the products appear well tolerated, and gastrointestinal side effects are the most prominent. However, a systematic review of cannabinoids (typically CBD plus THC) for pain, nausea and spasticity reported that the number needed to harm' was between 8 and 22 . Central effects were most common (dizziness, sedation confusion and dissociation), and 35\%-70\% reported 'feeling high'. Epidiolex is $99 \%$ CBD (0.1\% THC) in contrast to the oromucosal spray Sativex (nabiximols), which is a 50/50 mixture of the two. The FDA has approved two further cannabinoid drugs: Syndros or Marinol (dronabinol) to treat anorexia 
in patients with AIDS and chemotherapy-associated nausea; and Cesamet (nabilone) again for intractable chemotherapy nausea. Dronabinol is a gelatine capsule containing delta-9-tetrahydrocannabinol while nabilone is a synthetic cannabinoid that acts like THC.

\section{THE STATE OF PLAY}

In the UK, CBD is being studied at major paediatric neurology centres such as Muir Maxwell Epilepsy Centre in Edinburgh, Great Ormond Street Hospital in London, The Royal Hospital for Sick Children in Glasgow and Alder Hey Children's Hospital in Liverpool. Studies began in 2014 and as such there is a growing clinical experience with Epidiolex. Trials were initially in Dravet syndrome, and some of these children may have transitioned into adult neurology over time.

CBD trials reporting over the last two years have successfully transitioned the position of the drug from 'anecdotal and promising' to 'proven to be effective'. Well-designed placebo-controlled trials in patient groups who are not normally recruited to epilepsy drug studies (children and people with intellectual disability) have established the benefit of Epidiolex in Dravet and Lennox-Gastaut syndromes. ${ }^{3-5}$ The study in Dravet syndrome recruited 120 children and young adults, whereas the Lennox-Gastaut papers randomised 225 children and 171 young adults. Interesting points include the $5 \%$ of patients with Dravet syndrome who became seizure free (none on placebo); seizure freedom in Dravet syndrome is very rare. Both Lennox-Gastaut syndrome papers focused on drop seizures, which are disabling and injurious. The Devinksy et al paper ${ }^{4}$ randomised to three groups, allowing comparison of 10 and $20 \mathrm{mg}$ doses; both active arms saw a significant reduction in drop seizures compared with placebo.

Australia, Argentina, Canada, Colombia, the Czech Republic, Denmark, Finland, Germany, Greece, Israel, Italy, the Netherlands, Poland, Switzerland, Uruguay and Zimbabwe are among the nations where medicinal cannabis-derived drugs are legal. Cannabis remains a Schedule I drug in most parts of the USA, despite a movement to legalise cannabis and cannabis-derived drugs in many states. Schedule I drugs are defined as drugs with no currently accepted medical use and a high potential for abuse; including heroin, LSD and ecstasy. Hemp-derived CBD is legal in all 50 states, whereas marijuana-CBD (more likely to be THC-rich) is legal for medicinal purposes in 46 states and legal for recreational purposes in 8 of those states without a prescription; namely Alaska, California, Colorado, Maine, Massachusetts, Nevada, Oregon and Washington. In the UK, cannabis is a class B drug, which means that a criminal conviction for producing and supplying the drug could be punished with a maximum of 14 years in prison, an unlimited fine or both. This is a precarious situation. If the individual wants to travel abroad with a cannabis-derived drug, they would need to investigate intensively the legal status of the compounds in the country they wish to visit; in the same way that codeine or clobazam need to be considered.

Cannabis is a Schedule I drug in the UK within the Misuse of Drugs Regulations 2001 (pertaining to lawful possession and supply) ${ }^{6}$ but in July 2018 the Advisory Council on the Misuse of Drugs recommended it should be downgraded to Schedule II, in theory allowing clinicians to prescribe cannabis. However, CBD that is devoid of THC is classified as Schedule IV; Sativex, despite its THC, has special dispensation to be Schedule IV.

Each nation's approach to CBD has been different. In Denmark, for example, cannabis-derived products (including Sativex) were legalised for a 4-year trial period from 1 January 2018. This opened the door to imported products and to those manufactured outside of traditional pharmaceutical processes. This meant that the Danes had to cope with certain practical issues ahead of many other nations. Their approach to driving eligibility was to state that anyone taking it must respect a complete driving ban-although they immediately softened this stance by allowing a clinician's discretion to be used, providing that there was no discernible THC in the preparation used. Also, clinicians had to learn quickly how best to advise and dose people who wanted their cannabis-derived products in non-standard ways-such as in tea-like infusions. The primary indications for cannabis-deprived products in Denmark have been multiple sclerosis and nausea and vomiting in palliative care.

There are currently 151 clinical trials listed on clinicaltrials.gov, the American (NIH) repository of studies, 21 of which relate to epilepsy. There are ongoing trials for cancer pain, schizophrenia and new epilepsy indications. A recent systematic review confirms that CBD is more effective than placebo at reducing seizure burden $(50 \%$ seizure reduction $)$-with a relative risk of 1.7-2.4, and a number needed to treat of $8 .^{357}$ Across 12 pooled observational studies there was a $56 \%$ increase in quality of life, a $51 \%$ rate of adverse events and a $2.2 \%$ risk of serious adverse events. They noted that there was more reliance than usual on observational studies than randomised controlled trials; extension studies are less likely to feature patients who cannot tolerate the drug.

On 19 April 2018 the FDA published a briefing document following a request from GW Pharmaceuticals to appraise the evidence behind CBD for treating seizures in Lennox-Gastaut and Dravet syndromes. ${ }^{1}$ They were able to appraise 1391 people who had taken CBD for epilepsy. The median exposure was 275 days and the maximum 1025. There have been 20 deaths reported-most appear to have been late, rather than early or immediately after receiving the drug. One was during the $20 \mathrm{mg} / \mathrm{kg}$ trial phase, 7 in the open-label 
extension and 12 in the expanded access programme. There were no deaths with placebo. Although many of the deaths could be attributed to the progression of the underlying disease (mitochondrial disorder and Batten's disease were mentioned), eight were either respiratory in nature or described as a SUDEP. This is important because CBD has a clinically relevant effect in potentiating clobazam. The FDA report considered the documented rise in serum transaminase as a clear drug effect-seen in $4 \%$ of cases but $0 \%$ placebo, although there were no cases of true hepatic failure. In a study of patients in the extended access programme, $75 \%$ of those with significant increases in liver enzymes were co-prescribed valproate. ${ }^{8}$ The FDA also drew reference to the infection rate-pneumonia in $4 \%$ of cases ( $0 \%$ placebo) and somnolence $2 \%$ of cases ( $0 \%$ placebo). The pneumonia risk was notably 9.1 times higher in those receiving CBD.

Finally, there are open-label studies being published beyond Epidiolex. Tilray 2:100 is a mixed THC/CBD compound containing $2 \mathrm{mg} / \mathrm{mL}$ THC and $100 \mathrm{mg} / \mathrm{mL}$ CBD. In a study aiming to establish dosing and tolerability, 20 children with Dravet were trialled on this drug for 20 weeks. ${ }^{9}$ Even in such a small study there were reported significant improvements in quality of life, and a reduction in motor seizures of $71 \%$, with a $50 \%$ responder rate of $63 \%$.

\section{WHAT DO PATIENTS NEED TO KNOW?}

New medications are desperately needed as $30 \%-40 \%$ of people with epilepsy do not respond to traditional medication regimens. ${ }^{10}$ This is more likely when the person with epilepsy has additional needs such as autism and intellectual disability. There is a great deal of concern from patients and their families about the long-term effects of chronic antiepilepsy drug use. Much of this is well founded with drug-specific concerns such as retigabine causing blue staining of skin and retinal pigment, ${ }^{11}$ and drugs such as carbamazepine being associated with osteoporosis and accelerated atherosclerosis. ${ }^{12}$ Others are concerns seen across most antiepilepsy drugs such as the neurodevelopmental consequences of valproate exposure in utero (but also other antiepilepsy drugs at high doses or at polytherapy) or the generalised and cerebellar atrophy associated with chronic drug exposure.

Not all drugs are designed; some are repurposed and some are serendipitous accidents. There is a desire for more 'natural' - and less 'toxic' medications. Unfortunately, there is a strong correlation in the public consciousness with plant-based medications and a supposition that these have milder side effects and make safer drugs. The pharmaceutical companies do nothing to dispel this misinformation by promoting their industrial agriculture plants. Aspirin is a wonder drug-and digoxin has undoubtedly helped millions-but both are characterised by the presence of their adverse side effects and drug interactions rather than the lack of them. Cyanide, of course, is a natural drug. That CBD is derived from the street drug cannabis - and yet still patients equate the drug with safety-speaks to how cannabis use has broken into the mainstream of Western culture, and how safe many people must see this illegal substance.

\section{The desire for this to work}

We have a major issue with the placebo effect in clinical epilepsy and in epilepsy drug trials. ${ }^{13}$ This is in part because we have no biomarker for seizure frequency or for epilepsy control. If only we had an equivalent of the HbA1c used in diabetes that we could use for epilepsy. The second factor is that for eligibility to enter a trial we often require patients to demonstrate a high seizure load in the weeks before the study starts. This may not be their habitual seizure frequency and, as a result, often doing nothing would allow their seizures to be become less frequent: the phenomenon of returning to the mean. We also risk this when changing medication at times of crisis. Too often patients talk about a drug having a 'honeymoon' effect-by which they mean, when a new drug was started it seemed to work-but then the seizures returned. Anecdotally, however, we have been told of parents coming back to clinic and blaming themselves rather than CBD for its failureso strong is their conviction that this drug will work for their child. The placebo response to the drug is high and the parents have been slow to report negative side effects. I have certainly had more patients speak to me openly about their chronic cannabis use recentlyoften with the expectation that I may suggest that it should continue for its medical benefits.

\section{Vulnerability}

Patients are remarkably vulnerable currently because cannabis products including artisanal cannabis oils-at variable concentrations-are available at high-street retailers such as Holland \& Barrett, from independent retailers (so-called head shops) and via online sources (figure 1). Interestingly, some high street retailers stock the oil under 'Homeopathic and Flower remedies'. We currently have no independent way to quantify for our patients what is in these substances. It is important to state that these are not pharmaceutical-grade products. Moreover, we cannot advise about sources, doses or regimens. A community-based study of patients supplying their own CBD showed that doses reported by parents ranged from 0.5 to $28.6 \mathrm{mg} / \mathrm{kg} /$ day, and THC dosages ranged from 0 to $0.8 \mathrm{mg} / \mathrm{kg} / \mathrm{day} .{ }^{14}$ If patients are frank with us that they are taking these supplements, then we may choose to monitor serum antiepilepsy drug concentrations for clinically relevant drug interactions. It is likely that all available CBD is subtherapeutic, but still problematic. CBD does not readily cross the blood-brain barrier and therefore it is possible to have peripheral effects and drug-drug interactions without the required central effects. 


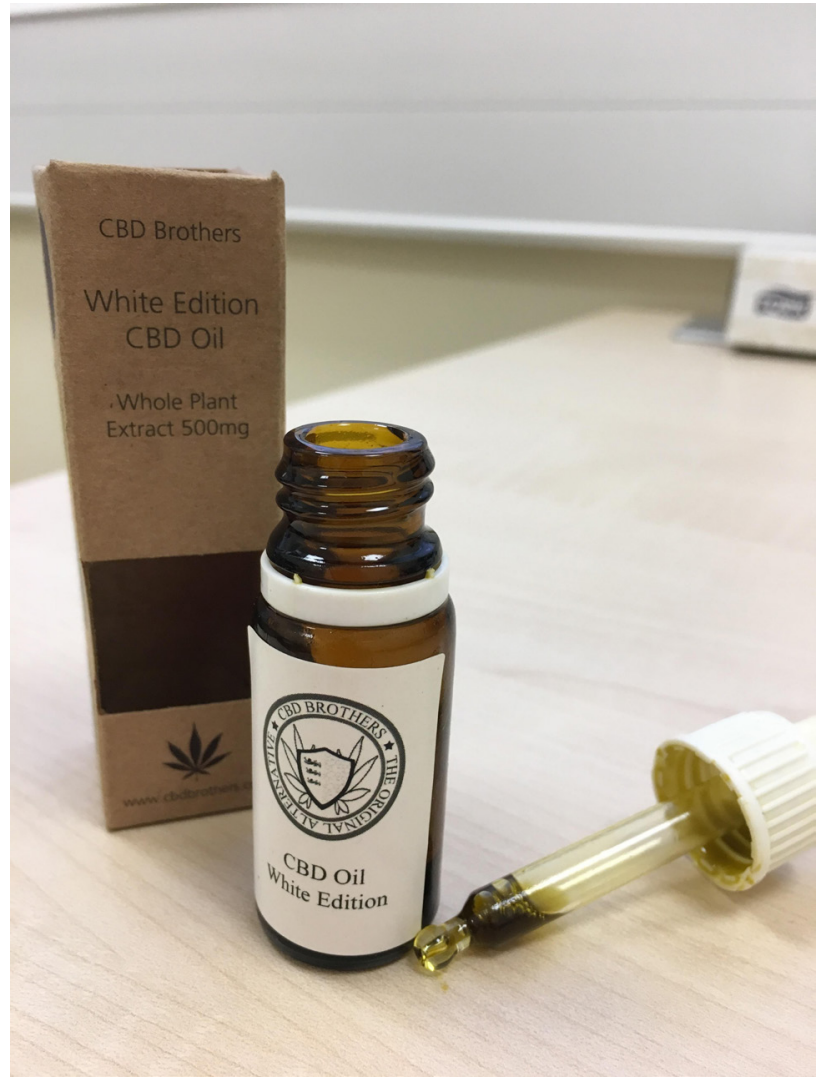

Figure 1 Artisanal cannabidiol (CBD) is sold as a dietary supplement. A patient shows me their CBD oil bought on line and we have no way to assay the contents of the agent.

Often these patients are on a clinical knife edge and are hospitalised with seizures from time to time. We cannot ask our nursing staff or pharmacists to handle these substances (literally or metaphorically), and therefore if patients were to continue them, their parents would need to administer every dose. Similarly, until legalised, tolerated or prescribable, patients on named-patient basis-only schedules are at risk of a Home Office volte face and the removal of their drug. There are political and economic groups that support the legalisation of cannabis who will seek to exploit individual cases for the greater benefit of their cause. There is no limit to what advocates have claimed medical cannabis can cure, but the failures are never publicised (figure 2). Medical cannabis has been cleverly marketed and has captured the imagination of much of the population.

\section{Access to CBD}

The UK press reporting of epilepsy and CBD shows much confusion. Artisanal CBD available on the high street is conflated with highly effective medications that have strong trial evidence. Little distinction is made between products with low THC content trialled in the UK, and CBD compounds where there is a $>0.2 \%$ THC content-which would be currently illegal in the UK. The situation is changing quickly and the Home
Office has announced the creation of an Expert Panel that now has a published membership and terms of reference. ${ }^{15}$ At time of writing, the process of application and access to prescriptions is opaque. Patients and clinicians alike are confused by headlines such as 'Cannabis-based medicines get green light as UK eases rules' (figure 3) (Guardian 26 July 2018).

\section{WHAT IT WOULD BE NICE TO KNOW}

How does CBD work? One would imagine that CBD would exert its effect via an interaction with cannabinoid receptors-but this is not thought to be the case and indeed we do not know the exact mechanism of the antiepileptic effect of CBD. We do know that CBD acts at multiple sites, which include intracellular targets such as mitochondria ${ }^{16}$ and targets located on neuronal membranes-ion channels (voltage-gated sodium channels, voltage-gated calcium channels), neurotransmitter receptors (GABA, 5-HT) and G-protein coupled receptors (GPR55). The ability of CBD to exert symptomatic benefits via multiple mechanisms may represent a novel poly-pharmacological approach to the disease. In a comprehensive review, Brodie et $a l^{17}$ made a compelling case for how CBD could exert a multitarget impact via (1) inhibition of voltage-gated sodium channels; (2) activation and desensitisation of transient receptor potential cation (TRPV1) channels; (3) inhibition of rapamycin signalling; (4) direct modulation of 5-HT signalling; (5) indirect activation of adenosine and (6) the general inhibition of neuroinflammation. The CB1 receptor is where THC acts, whereas $\mathrm{CB} 2$ is the target for cannabinol. CBD has been hypothesised to have neuroprotective and anti-inflammatory effects; both of which would be attractive traits in an anti-epilepsy drug. This broad mode of action may well be a benefit rather than a curse-many of the most effective anti-epilepsy drugs, valproate for example, have diverse mechanisms of action.

What is the importance of serum THC concentrations in the cannabis-derived product for the control of seizures? There is no clinical evidence. Rosenberg et $a l^{18}$ reviewed the preclinical literature and reported that across 34 studies using six disparate animal species, THC demonstrated an anticonvulsant action $(62 \%)$, proconvulsant $(3 \%)$, mixed $(3 \%)$ and no effect $(32 \%)$ in seizure models. In the rodent maximal electroshock model of generalised seizures, THC was capable of increasing the effects of phenytoin and phenobarbital. ${ }^{19}{ }^{20}$ In addition to the $\mathrm{CB}_{1} / \mathrm{CB}_{2}$ receptor-related effects of THC on neuronal excitability ${ }^{18}$ and neurotransmitter release, THC is also likely to influence seizure activity via anti-inflammatory ${ }^{21}$ and antioxidant $\mathrm{t}^{22}$ actions. In addition, similar to CBD, there is evidence for THC's ability transiently to activate and desensitise transient receptor potential (TRPA1, TRPV1 and TRPV2) channels. ${ }^{23-25}$ However, 

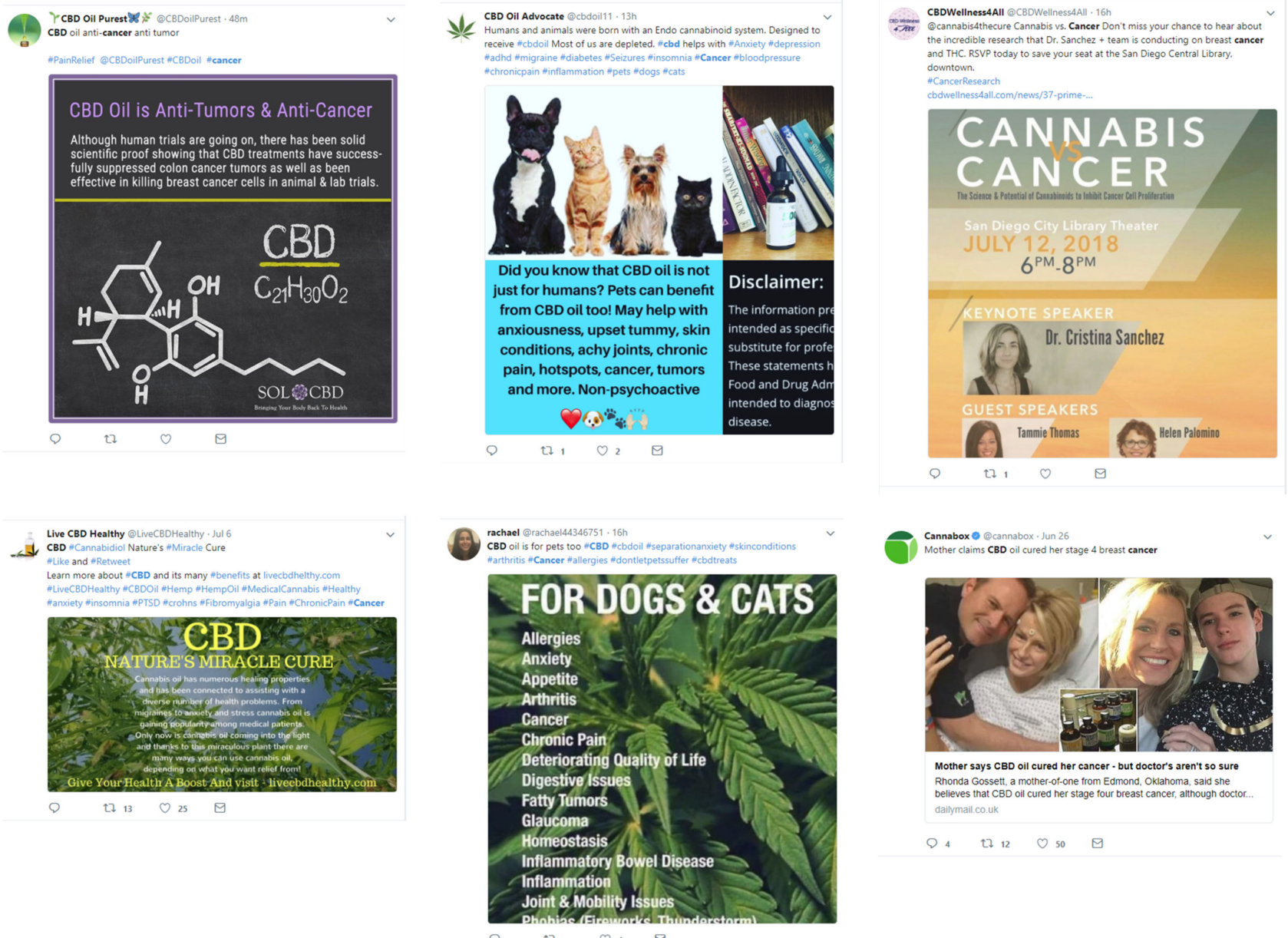

Figure $2 \mathrm{~N}=1$ trials and the power of anecdote. Social media claims regarding cannabidiol (CBD) and cancer (12 July 2018). All claims regarding the treatment of cancer would likely contravene the Advertising Standards Agency requirements in the UK.

the consequences of high-dose THC on the juvenile and still-developing brain are currently unknown.

CBD and clobazam have bi-directional and a clinically relevant drug interaction. This is because CBD inhibits cytochrome P450 (CYP)2C19 and increases serum concentrations of the clobazam metabolite, nordesmethyl. In the recently published data from the CBD extended-access programme, 59\% of patients reduced their baseline clobazam, 50\% reduced their baseline valproate and $21 \%$ their levetiracetam. The rate of reported somnolence was 2.7 times more likely in patients who were co-prescribed clobazam. ${ }^{8}$

Does CBD produce most of its effect by potentiating the effects of clobazam-should there be a co-drug? This theory may be supported by recent evidence which shows that $\mathrm{CBD}$ acts as a positive allosteric modulator at the $\mathrm{GABA}_{\mathrm{A}}$ receptor. ${ }^{26}$ This finding raises the possibility that combined dosing of clobazam and CBD may produce a synergistic augmentation of $\mathrm{GABA}_{\mathrm{A}}$ receptors and enhanced seizure control. Demonstration of synergism requires use of specific methods of analysis that include isobologram analysis. Future well-designed preclinical studies using such approaches would be critical to support this hypothesis of synergy with, if positive, subsequent translation to rigorous clinical trials.

You could ask what would be the risks associated with immediately licensing CBD and cannabis-derived products for all people with epilepsy? We fear that the regulatory trials would not be completed. We would be less likely to have open reports from phase IV extension studies, but adverse effects would still be yellow-carded. This might be very much like our current situation with the ketogenic diet. We would not have guidance as to who best would respond to CBD. The ketogenic diet has crept slowly from paediatric to adults practice and is extending out now to neuro-oncological studies because drug companies are not bank-rolling further trials. We could be left with the strong trial evidence in Dravet and LennoxGastaut syndromes, but the evidence may not be well escalated beyond people with multiple seizure types that include myoclonic seizures. It would be a mistake to rush to license CBD and cannabis derivative products without completing the therapeutic licensing process. 


\section{Cannabis-based medicines get green light as UK eases rules}

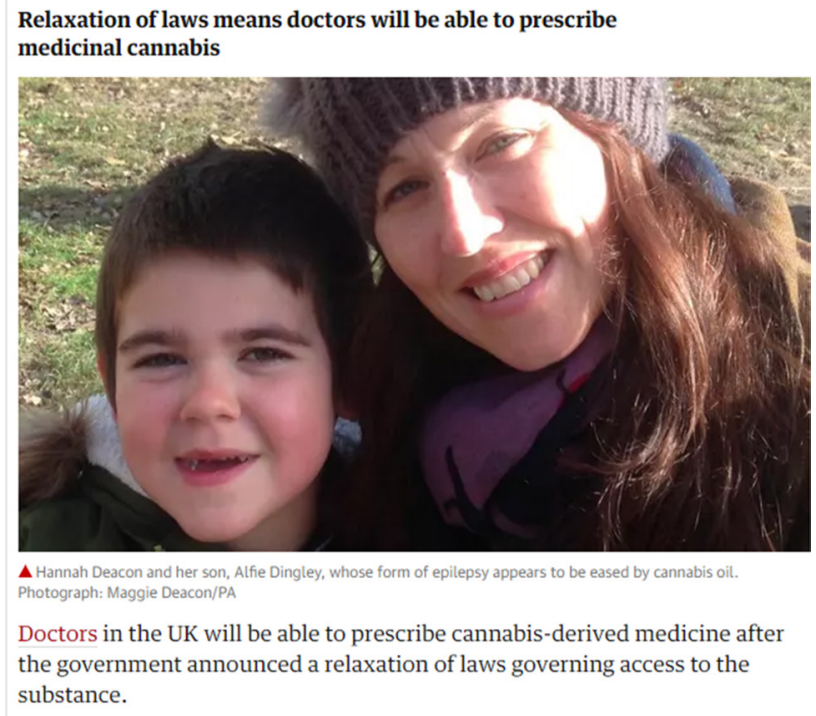

Figure 3 Cannabidiol (CBD) in the press. A new application process has been announced but families are very confused about the availability of medicinal-grade CBD. There needs to be rigorous regulatory and monitoring processes around this scheme.

\section{WHAT DO NEUROLOGISTS NEED TO KNOW?}

1. We need to be better at identifying and diagnosing Lennox-Gastaut and Dravet syndromes. Dravet is common at an estimated incidence of 1 in 20000 births. $^{27}$ We advise improving links with paediatric neurology, and having more enthusiasm for epilepsy gene panel testing and 'medical archaeology' (digging in to the old notes).

2. CBD is not the only treatment for people with Dravet syndrome; stiripentol and fenfluramine are both effective and published clinical data confirm their safety profile and longer-term efficacy. ${ }^{28} 29$

3. We need to be alert to new drug interactions: do we need to be testing serum concentrations of clobazam?

4. People will be 'taking matters in to their own hands' and there may be an upswing in the recreational use of cannabis in an attempt to help their seizure control.

5. There are many unanswered questions. Are people who are seizure free but taking cannabis-derived medication safe to drive? How do we counsel women who may become pregnant?

We are excited about the possibilities of this new agent and excited that patients appear to want to trial this product. As medics, we should divorce our thoughts about artisanal CBD oils from the CBD rigorously tested in clinical trials. CBD is not a panacea, but nor is it our first step towards inevitable societal doom. The arguments about who may benefit from cannabis-derived medicines should be entirely uncoupled from discussion surrounding the legalisation of recreational cannabis.

\section{Key points}

We should divorce our thoughts about artisanal cannabidiol oils from the cannabidiol rigorously tested in clinical trials.

- Cannabidiol is not a panacea, but nor is it our first step towards inevitable societal doom.

- The arguments about who may benefit from cannabisderived medicines should be entirely uncoupled from discussion surrounding the legalisation of recreational cannabis.

- Cannabidiol medications may not primarily work on cannabidiol receptors and may have significant interactions with clobazam.

Contributors RHT and MOC contributed equally to the design, drafting and development of this review.

Funding The authors have not declared a specific grant for this research from any funding agency in the public, commercial or not-for-profit sectors.

Competing interests RHT receives speaker fees from Eisai Ltd and UCB Pharma, he has consulted for Sanofi and Zogenix. RHT is an associate Editor of Practical Neurology. MOC has consulted for Neurexpert and Eisai Ltd. MOC has active scientific collaborations with Takeda, Eisai and Autifony Therapeutics. MOC has scientific collaborations with GW Pharma and GSK.

Patient consent Not required.

Provenance and peer review Commissioned. Externally peer reviewed by Helen Cross, London, UK.

\section{REFERENCES}

1 Peripheral and Central Nervous System Drugs Advisory Committee Meeting, 2018. FDA briefing document. https:// fda.gov/downloads/advisorycommittees/committeesmeetingmat erials/drugs/peripheralandcentralnervoussystemdrugsadviso rycommittee/ucm604736.pdf (accessed 21 Jul 2018).

2 Allan GM, Finley CR, Ton J, et al. Systematic review of systematic reviews for medical cannabinoids: Pain, nausea and vomiting, spasticity, and harms. Can Fam Physician 2018;64:e78-e94.

3 Devinsky O, Cross JH, Laux L. Cannabidiol in dravet syndrome study groDravet Syndrome Study Group. Trial of cannabidiol for drug-resistant seizurCannabidiol for DrugResistant Seizures in the dravet synDravet Syndrome. N Engl J Med 2017;376:2011-20.

4 Devinsky O, Patel AD, Cross JH, et al. Effect of cannabidiol on drop seizures in the lennox-gastaut syndrome. N Engl J Med 2018;378:1888-97.

5 Thiele EA, Marsh ED, French JA, et al. Cannabidiol in patients with seizures associated with lennox-gastaut syndrome (GWPCARE4): a randomised, double-blind, placebocontrolled phase 3 trial. Lancet 2018;391:1085-96.

6 legislation.gov.uk, 2001. The misuse of drugs regulations. http://www.legislation.gov.uk/uksi/2001/3998/schedule/1/made (accessed 16 Aug 2018).

7 Stockings E, Zagic D, Campbell G, et al. Evidence for cannabis and cannabinoids for epilepsy: a systematic review of controlled and observational evidence. J Neurol Neurosurg Psychiatry 2018;89:741-53. 
8 Szaflarski JP, Bebin EM, Comi AM, et al. Long-term safety and treatment effects of cannabidiol in children and adults with treatment-resistant epilepsies: Expanded access program results. Epilepsia 2018;59:1540-8.

9 McCoy B, Wang L, Zak M, et al. A prospective open-label trial of a CBD/THC cannabis oil in dravet syndrome. Ann Clin Transl Neurol 2018;5:1077-88.

10 Chen Z, Brodie MJ, Liew D, et al. Treatment outcomes in patients with newly diagnosed epilepsy treated with established and new antiepileptic drugs: a 30-year longitudinal cohort study. JAMA Neurol 2018;75:279-86.

11 Garin Shkolnik T, Feuerman H, Didkovsky E, et al. Bluegray mucocutaneous discoloration: a new adverse effect of ezogabine. JAMA Dermatol 2014;150:984-9.

12 Chuang YC, Chuang HY, Lin TK, et al. Effects of long-term antiepileptic drug monotherapy on vascular risk factors and atherosclerosis. Epilepsia 2012;53:120-8.

13 Goldenholz DM, Moss R, Scott J, et al. Confusing placebo effect with natural history in epilepsy: a big data approach. Ann Neurol 2015;78:329-36.

14 Porter BE, Jacobson C. Report of a parent survey of cannabidiol-enriched cannabis use in pediatric treatmentresistant epilepsy. Epilepsy Behav 2013;29:574-7.

15 Gov.uk, 2018. Cannabis and cannabis-based medicinal products. https://www.gov.uk/government/publications/ cannabis-based-medicines-expert-review-panel/cannabis-andcannabis-based-medicinal-products

16 Ryan D, Drysdale AJ, Lafourcade C, et al. Cannabidiol targets mitochondria to regulate intracellular Ca2+ levels. J Neurosci 2009;29:2053-63.

17 Brodie JS, Di Marzo V, Guy GW. Polypharmacology shakes hands with complex aetiopathology. Trends Pharmacol Sci 2015;36:802-21.

18 Rosenberg EC, Tsien RW, Whalley BJ, et al. Cannabinoids and epilepsy. Neurotherapeutics 2015;12:747-68.
19 Chesher GB, Jackson DM. The effect of withdrawal from cannabis on pentylenetetrazol convulsive threshold in mice. Psychopharmacologia 1974;40:129-35.

20 Chesher GB, Jackson DM, Malor RM. Interaction of delta9tetrahydrocannabinol and cannabidiol with phenobarbitone in protecting mice from electrically induced convulsions. J Pharm Pharmacol 1975;27:608-9.

21 Benito C, Tolón RM, Pazos MR, et al. Cannabinoid CB2 receptors in human brain inflammation. Br J Pharmacol 2008;153:277-85.

22 Hampson AJ, Grimaldi M, Axelrod J, et al. Cannabidiol and (-) Delta9-tetrahydrocannabinol are neuroprotective antioxidants. Proc Natl Acad Sci U S A 1998;95:8268-73.

23 De Petrocellis L, Vellani V, Schiano-Moriello A, et al. Plantderived cannabinoids modulate the activity of transient receptor potential channels of ankyrin type- 1 and melastatin type-8. J Pharmacol Exp Ther 2008;325:1007-15.

24 De Petrocellis L, Ligresti A, Moriello AS, et al. Effects of cannabinoids and cannabinoid-enriched Cannabis extracts on TRP channels and endocannabinoid metabolic enzymes. $\mathrm{Br} J$ Pharmacol 2011;163:1479-94.

25 Qin N, Neeper MP, Liu Y, et al. TRPV2 is activated by cannabidiol and mediates CGRP release in cultured rat dorsal root ganglion neurons. J Neurosci 2008;28:6231-8.

26 Bakas T, van Nieuwenhuijzen PS, Devenish SO, et al. The direct actions of cannabidiol and 2-arachidonoyl glycerol at $\mathrm{GAB}_{\mathrm{AA}}$ receptors. Pharmacol Res 2017;119:358-70.

27 Scheffer IE. Diagnosis and long-term course of Dravet syndrome. Eur J Paediatr Neurol 2012;16 Suppl 1:S5-S8.

28 Myers KA, Lightfoot P, Patil SG, et al. Stiripentol efficacy and safety in Dravet syndrome: a 12-year observational study. Dev Med Child Neurol 2018;60:574-8.

29 Ceulemans B, Schoonjans AS, Marchau F, et al. Five-year extended follow-up status of 10 patients with Dravet syndrome treated with fenfluramine. Epilepsia 2016;57:e129-e134. 